\title{
MITIGATING DISCONTINUITIES IN SEGMENTED KARHUNEN-LOÈVE TRANSFORMS
}

\author{
Monika Stadnicka ${ }^{\dagger}$ Ian Blanes ${ }^{\dagger}$, Joan Serra-Sagristà ${ }^{\dagger}$, Michael W. Marcellin ${ }^{\ddagger}$ \\ ${ }^{\dagger}$ Department of Information and Communications Eng., Universitat Autònoma de Barcelona, Spain \\ ${ }^{\ddagger}$ Department of Electrical and Computer Eng., University of Arizona, USA
}

\begin{abstract}
The Karhunen-Loève Transform (KLT) is a popular transform used in multiple image processing scenarios. Sometimes, the application of the KLT is not carried out as a single transform over an entire image. Rather, the image is divided into smaller spatial regions (segments), each of which is transformed by a smaller dimensional KLT. Such a situation may penalize the transform efficiency. An improvement for the segmented KLT, aiming at mitigating discontinuities arising on the edge of adjacent regions, is proposed in this paper. In the case of moderately varying image regions, discontinuities occur as the consequence of disregarded similarity between transform domains, as the order and sign of eigenvectors in the transform matrices are mismatched. In the proposed method, the KLT is adjusted to guarantee the best achievable similarity via the optimal assignment and sign correspondence for eigenvectors. Experimental results indicate that the proposed transform improves the similarity between transform domains, and reduces RMSE on the edge of adjacent regions. In consequence, images processed by the adjusted KLT present better cohesion and continuity between independently transformed regions.
\end{abstract}

Index Terms - Karhunen-Loève Transform, KLT, Segmentation, Discontinuities, Hyperspectral Images

\section{INTRODUCTION}

The Karhunen-Loève Transform (KLT) is widely employed by the image processing community, both for analysis and for compression. Its field of application is vast and it has received many names over the years, such as Principal Component Analysis (PCA), Hotelling transform, spectral decomposition, etc. The KLT is an orthogonal transform that provides optimal data decorrelation, as well as optimal energy compaction [1].

Being a data-dependent transform, the KLT needs to be trained for a given image before it can be applied. In the case of a segmented application of the KLT, distinct image regions are transformed (and trained) independently. Such segmented

This work was supported in part by FEDER, the Spanish Government (MINECO), and the Catalan Government, under grants TIN2015-71126-R and 2014SGR-691. application requires less memory since a smaller portion of the image needs to be buffered while the transform is trained. Image segmentation not only improves the transform feasibility, but may provide some improvements in analysis and compression performance $[2,3]$.

Hyperspectral images -i.e., those images encompassing hundreds of image components, each corresponding to a different wavelength - are one particular case where a segmented KLT is of frequent use. Even though the applicability of the proposed work is not restricted to hyperspectral images, the scope presented here has been narrowed down to this case, in part because of the following use case. It is well known that space-borne equipment requires efficient data compression techniques due to constraints in the downlink channel capacity and the use of radiation hardened hardware [4-6]. In particular, the Consultative Committee for Space Data Systems (CCSDS) [7] —a consortium of major space agenciesis currently preparing a new lossy transform coding standard for hyperspectral images, in which a segmented KLT may be employed to alleviate the memory buffering needs of an unsegmented KLT.

Segmented application of the KLT has been studied on multiple occasions. Hyperspectral images can be segmented based on spectral signature, such as in $[2,8]$, where a global KLT is employed to distinguish spectrally similar regions before applying a KLT for each region independently. In $[9,10]$ segmentation is achieved through Vector Quantization, followed by independent KLTs.

Segmentation can also be performed according to spatial proximity. In [11] a Bayesian-based segmentation algorithm is considered as an alternative to global image processing. In [12] the problem of arbitrarily shaped image segments is considered, suggesting disjunctive coding of rectangular blocks and sparse pixels. The issue addressed in [13] is the segmentation problem using neural networks. The concept of weighted KLT and overlapping blocks is proposed in $[14,15]$. While [14] strives to reduce blocking artifacts, [15] employs a lapped transform for the purpose of computational complexity reduction and better coding performance. Overviews of different segmentation methods and coding techniques are found in $[3,16,17]$, where a spatially compact segmentation followed by an adaptive KLT is revealed as an efficient coding scheme. 
A desirable feature for a segmented KLT is that when input regions are similar, transformed outputs are consistently similar. Such consistency would allow exploitation of shared statistics of multiple KLTs by, for example, better preserving a complex entropy model between adjacent transformed data. As this could not be guaranteed, authors have had to work around this issue $[2,9,10]$ or leave out the KLT due to the discontinuities [18].

In this paper the problem of the discontinuities in a segmented KLT is studied and a method is proposed to improve image continuity in the transformed domain. Section 2 presents the source of discontinuities, introduces the problem formulation and explains its complexity. A discontinuity mitigation strategy is presented in Section 3. Experimental results are discussed in Section 4. The last section draws some conclusions.

\section{THE PROBLEM OF DISCONTINUITIES}

The KLT may be understood as a transform that changes the coordinate system for a given cloud of multidimensional samples, i.e., each sample having multiple features. Let $X$ be a data matrix that has samples for columns and features for rows. Then, $Y$, the outcome after applying the transform, is computed as

$$
Y=\operatorname{KLT}_{\Sigma_{X}}(X)=Q^{T} X,
$$

where $\Sigma_{X}$ is the covariance matrix of the rows of $X$, and $Q$ is the orthogonal matrix obtained from the eigendecomposition of

$$
\Sigma_{X}=Q \Lambda Q^{-1} .
$$

The transform matrix $Q$, given as the solution to the eigendecomposition problem in (2), is invariant to two operations: first, the eigenvectors (columns of $Q$ ) can be permuted arbitrarily, and second, their signs can be freely changed. None of these operations violates the requirements of the eigendecomposition, and consequently, $Q$ is not unique. In general, given a transform matrix $Q$, equivalent transform matrices can be found as

$$
Q^{\prime}=Q \cdot P \cdot S,
$$

where $S$ is a diagonal matrix with non-zero elements \pm 1 , and $P$ is a permutation matrix. That is, $P$ is a matrix having a single 1 in each row and each column. This matrix serves to permute the columns of $Q$.

A canonical solution to the eigendecomposition problem is commonly selected, such as the one that has eigenvectors arranged by decreasing magnitude of their associated eigenvalues (choice of $P$ ) and where the first non-zero element of each eigenvector is positive (choice of $S$ ). However, this presents a problem whenever the data samples are segmented and multiple KLTs are employed. In particular, this canonical solution occasionally leads to the occurrence of discontinuities between adjacent KLTs. For example, two segments may

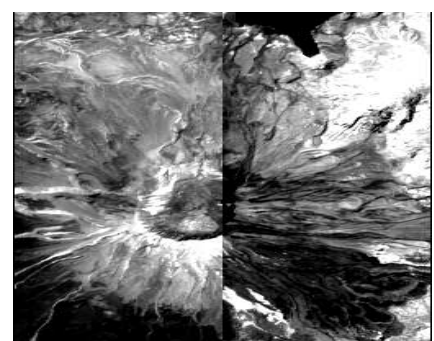

(a) Band 1, rows 1000-1399

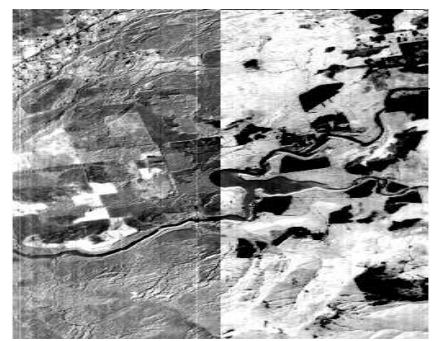

(b) Band 3, rows 0-399
Fig. 1: Independent conventional KLTs are applied on both sides of the Hyperion image EO1H0460282009231110KF, resulting in a discontinuity between regions.

have similar covariance matrices leading to similar eigenvectors and eigenvalues. Some of the eigenvalues within one decomposition may be similar in magnitude. In this case, even small variations in data between the two segments may alter the order of the eigenvalue magnitudes, thus changing the canonical order of the eigenvectors.

To illustrate this situation, an example on satellite data is provided in Fig. 1. In this figure, each pixel of a hyperspectral image was treated as a vector having dimension equal to the number of spectral bands. A one-dimensional KLT was designed and applied to each pixel in the left half of the image. A separate KLT was designed and applied to the right half of the image. Spatial crops from two different bands of the resulting transformed image are depicted in the figure. As can be seen, a very noticeable mismatch occurs between the left and right halfs for bands 1 and 3, due to the mismatching transforms designs.

From the discussion above, it is clear that discontinuities arise as the consequence of applying independent transforms to separate spatial regions within a multicomponent image. This manuscript takes into consideration only the two-region case. The case of a higher number of regions is intentionally left out of the scope of the paper due to space constraints. Nonetheless, note that multiple instances of the two-region case can be combined in scenarios where more than two KLTs are applied.

Given two regions for which two KLTs, $Q_{1}$ and $Q_{2}$, have been trained, discontinuities may result from when two regions are extremely different, resulting in extremely different $Q_{1}$ and $Q_{2}$. In this case, little can be done to improve cohesion. On the other hand, as discussed above, discontinuities may result from mismatching permutations and/or signs of the corresponding eigenvectors in $Q_{1}$ and $Q_{2}$. Without loss of generality, this can be mitigated by adjusting $Q_{2}$ to match $Q_{1}$ as

$$
Q_{2}^{\prime}=Q_{2} \cdot P \cdot S
$$

The best match between $Q_{1}$ and $Q_{2}^{\prime}$ is the one that provides the lowest divergence between the transform domains of the two KLTs. Suppose that a divergence $C$ can be com- 
puted to measure the mismatch between $Q_{1}$ and $Q_{2}^{\prime}$. Then, $C$ can be thought of as a function of $P$ and $S$. The problem to be addressed here can then be modeled as

$$
\underset{P, S}{\operatorname{argmin}} C(P, S) \text {. }
$$

Examining all possible combinations of $P$ and $S$ is not feasible. For data of $n$ dimensions (wavelengths or components), there are $n ! \cdot 2^{n}$ possible combinations of $P$ and $S$. To put that in perspective, for $n$ as little as 15 , this is over $10^{16}$ combinations, or more than 495 days testing a billion combinations per second.

\section{MITIGATION OF DISCONTINUITIES}

This section addresses discontinuities by minimizing their occurrence. In order to do so, two issues are investigated: first a measure of divergence is defined, and then the problem stated in (5) is solved.

Regarding the first issue, one may consider the scalar product of two normalized eigenvectors. Two equal eigenvectors have the maximum scalar product of 1.0 , which decreases as the vectors diverge ${ }^{1}$. Thus, the divergence between an eigenvector from $Q_{1}$ and the corresponding eigenvector from $Q_{2}^{\prime}$ can be taken as the negative of their scalar product. The divergence $C$ between the two transforms can then be taken as the (negative) sum of the scalar products between corresponding pairs of eigenvectors from $Q_{1}$ and $Q_{2}^{\prime}$. This can be formulated as

$$
\begin{aligned}
C(P, S) & =-\operatorname{tr}\left(Q_{1}^{T} \cdot Q_{2}^{\prime}\right) \\
& =-\operatorname{tr}\left(Q_{1}^{T} \cdot Q_{2} \cdot P \cdot S\right) .
\end{aligned}
$$

Regarding the second issue, the minimization problem defined in (5) can then be stated as

$$
\underset{P, S}{\operatorname{argmin}}-\operatorname{tr}\left(Q_{1}^{T} \cdot Q_{2} \cdot P \cdot S\right) .
$$

Disregarding $S$, this minimization problem can be modeled as a combinatorial problem called the assignment problem, originally represented by a set of workers (eigenvectors from $Q_{1}$ ) and a set of tasks to be done (eigenvectors from $Q_{2}$ ), where each task can be potentially done by each worker at a particular cost. The stated problem can be solved by the Hungarian algorithm [19], which takes as input a bi-adjacency matrix describing the cost of assigning each worker to each task, and yields an assignment of workers to tasks that minimizes the overall cost.

To take $S$ into account, the sign of an eigenvector from $Q_{2}$ is always chosen so that its scalar product with the target eigenvector from $Q_{1}$ is positive. The bi-adjacency matrix for the assignment problem then becomes

$$
\operatorname{abs}\left(Q_{1}^{T} \cdot Q_{2}\right),
$$

\footnotetext{
${ }^{1}$ The minimum scalar product of -1.0 occurs when the eigenvectors are collinear, but with opposite sign.
}

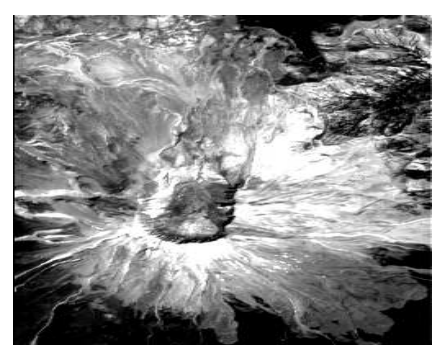

(a) Band 1, rows 1000-1399

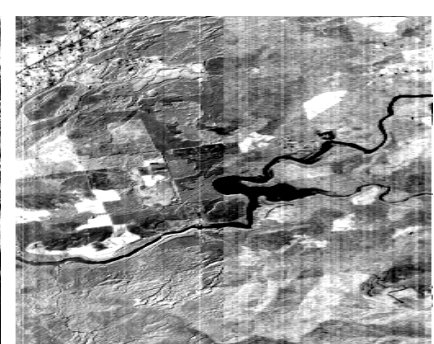

(b) Band 3, rows 0-399
Fig. 2: Independent adjusted KLTs are applied on both sides of the image. Due to the proposed mitigation strategy, the original discontinuities have disappeared.

and the assignment provided by the Hungarian algorithm yields $P$. Hereafter, the KLT with $P$ and $S$ selected in the manner described above is referred to as the adjusted KLT.

By modeling the discontinuity problem as an assignment problem and employing the scalar product as the basis of the measure of divergence, the problem can be efficiently solved in $O\left(n^{3}\right)$. It follows that the complexity of the adjusted KLT, as compared to the canonical KLT, is increased by at most a constant multiplicative factor since the training complexity of the KLT is already $O\left(n^{3}\right)$ [20].

\section{EXPERIMENTAL RESULTS}

In order to test the proposed method, a set of five hyperspectral images was used. This experimental set included two 242-band images obtained from the Hyperion sensor on board the EO-1 satellite (EO1H0240302009166110PF, EO1H0460282009231110KF) and three 224-band scenes obtained from Aviris Yellowstone (f060925t01p00r12_sc10, f060925t01p00r12_sc11 and f060925t01p00r12_sc18). In all experiments, the images were processed with the conventional (canonical) KLT, as well as the proposed adjusted KLT. The mean value in each region was subtracted prior to the application of either KLT. Fig. 2 provides a visual demonstration of the significant reduction in discontinuities provided by the adjusted KLT (compare with Fig. 1).

An objective measure of the effectiveness of the proposed method can be provided by the ratio of divergences $C_{A} / C_{C}$, where $C_{C}$ and $C_{A}$ are the divergences obtained with the conventional and adjusted KLT, respectively. The results of this calculation are depicted in Fig. 3. For each image, the number of transformed bands employed in the calculation of the ratio was varied. This is represented by the horizontal axis in the figure.

A $C_{A} / C_{C}$ ratio larger than 1 implies a lower divergence for the adjusted KLT. This can be seen by noting that divergence, as defined in (6), is negative. Thus, if $C_{A}$ is less (more negative) than $C_{C}$, the ratio will be larger than 1 . In general the adjusted KLT provides a significantly higher cohesion 


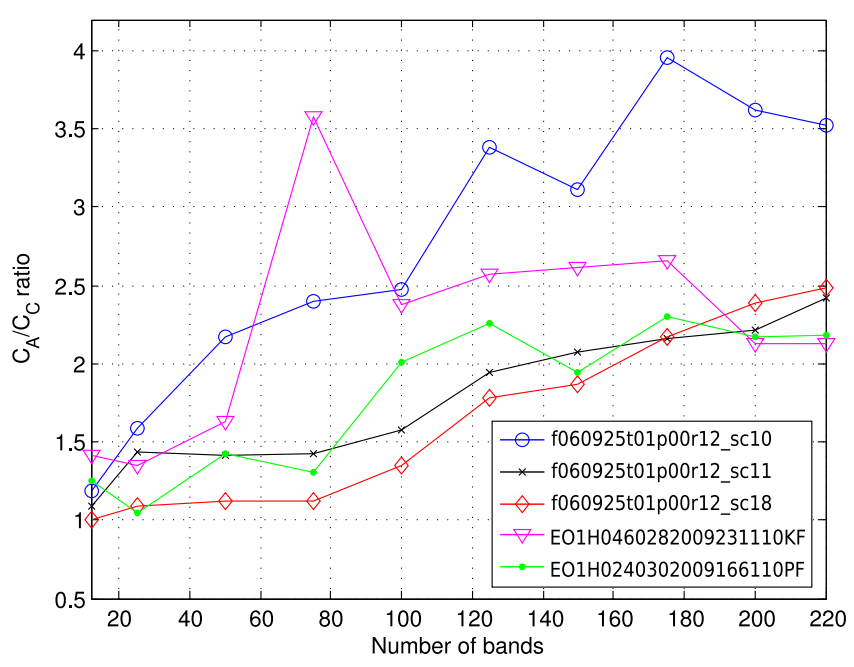

Fig. 3: Divergence ratio between adjusted and conventional KLT. The higher the ratio, the better the adjusted KLT performs over the conventional KLT.

between the two transform domains and, consequently, better continuity between regions. In particular, the figure suggests that divergence ratios up to 4.0 can be obtained. Moreover, the plot suggests that the $C_{A} / C_{C}$ ratio generally increases as the number of bands in the transformed images grows, demonstrating that the proposed method can be successfully applied to hyperspectral images comprising hundreds of bands.

In addition to the divergence ratio, RMSE along the boundary between image regions was computed as a measure of image consistency. In particular, for a given transformed band from a given image, let $y_{1}$ be the vector of pixels from the rightmost column of the left image region. Similarly, let $y_{2}$ be the vector of pixels from the leftmost column of the right image region. It is desirable that these vectors be as similar as possible. To this end, the RMSE between these two edge vectors was calculated for each band of each transformed image. This was carried out after processing with both the conventional and adjusted KLT. The results are presented in Fig. 4. Due to the scale, only results for the ten first bands are shown. For each band index (horizontal axis), the box-and-whisker plot on the left corresponds to the conventional KLT, while the box-and-whisker plot on the right corresponds to the adjusted KLT. Each box-and-whisker plot depicts the RMSE value for each of the five images.

As expected, the figure shows that images transformed with the conventional KLT tend to generate larger values of RMSE. Indeed, extreme values of RMSE occur in the initial bands, which could lead to significant problems for image analysis. These values are significantly reduced by the adjusted KLT. For example, in band 1 of the Hyperion image KF, the adjusted KLT reduces RMSE by more than $89 \%$. The relatively smaller RMSE that occurs in the images produced

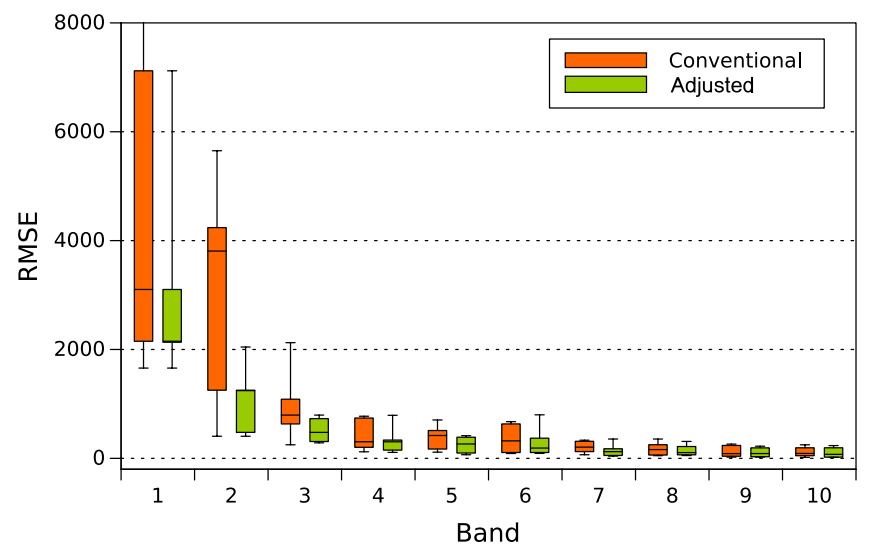

Fig. 4: Distribution of RMSE on the edge of adjacent image regions in the first ten bands of all images. Conventional and adjusted KLT are compared. Peak RMSE for the conventional KLT in band 1 is 19717.

by the adjusted KLT implies smoother transition between image segments and better preserved continuity.

\section{CONCLUSIONS}

In this paper, discontinuities resulting from a segmented KLT are studied. For a given image region, a KLT produces a transform matrix susceptible to permutations of eigenvectors and changes in eigenvector sign. On similar regions, discontinuities can result from these two operations on the KLT matrix. The discontinuity issue is modeled as a combinatorial minimization problem that can be reduced to the assignment problem, for which an efficient solution exists.

Experimental results suggest that the proposed method provides a significant improvement in similarity between adjacent transformed data and a notable diminution of RMSE on the edge of adjacent regions. The relative difference is most significant for the principal bands. The results also suggest that the efficacy increases as data dimensionality grows.

The proposed method effectively mitigates discontinuities that result from a segmented KLT, and does so without increasing the order of the computational cost of the KLT.

\section{REFERENCES}

[1] Y. Bandoh, H. Ohbayashi, S. Takamura, K. Kamikura, and Y. Yashima, "Mathematical analysis of the energy compaction affected by the dimensionality of KarhunenLoève transform," in Image Processing (ICIP), 2009 16th IEEE International Conference on, Nov 2009, pp. 21-24.

[2] G. Fernandez and C.M. Wittenbrink, "Coding of spectrally homogeneous regions in multispectral image compression," in Image Processing, 1996. Proceedings., In- 
ternational Conference on, Sep 1996, vol. 1, pp. 923926 vol.2.

[3] M. Cagnazzo, G. Poggi, and L. Verdoliva, "Regionbased transform coding of multispectral images," Image Processing, IEEE Transactions on, vol. 16, no. 12, pp. 2916-2926, Dec 2007.

[4] I. Blanes, E. Magli, and J. Serra-Sagrista, "A tutorial on image compression for optical space imaging systems," Geoscience and Remote Sensing Magazine, IEEE, vol. 2, no. 3, pp. 8-26, Sept 2014.

[5] I. Blanes and J. Serra-Sagristà, "Cost and scalability improvements to the Karhunen-Loève transform for remote-sensing image coding," Geoscience and Remote Sensing, IEEE Transactions on, vol. 48, no. 7, pp. 28542863, Jul. 2010.

[6] I. Blanes, M. Hernandez-Cabronero, F. Auli-Llinas, J. Serra-Sagrista, and M.W. Marcellin, "Isorange pairwise orthogonal transform," Geoscience and Remote Sensing, IEEE Transactions on, vol. 53, no. 6, pp. 33613372, June 2015.

[7] Consultative Committee for Space Data Systems (CCSDS), [Online]. Available: http://www.ccsds.org.

[8] G. Fernàndez and C. M. Wittenbrink, "Region based KLT for multispectral image compression," in European Signal Processing Conference, 1996. EUSIPCO 1996. 8th, Sept 1996, pp. 1-4.

[9] M. Finelli, G. Gelli, and G. Poggi, "Multispectral-image coding by spectral classification," in Image Processing, 1996. Proceedings., International Conference on, Sep 1996, vol. 1, pp. 605-608 vol.2.

[10] G. Gelli and G. Poggi, "Compression of multispectral images by spectral classification and transform coding," Image Processing, IEEE Transactions on, vol. 8, no. 4, pp. 476-489, Apr 1999.

[11] M. Cagnazzo, G. Poggi, and L. Verdoliva, "A comparison of flat and object-based transform coding techniques for the compression of multispectral images," in Image Processing, 2005. ICIP 2005. IEEE International Conference on, Sept 2005, vol. 1, pp. I-657-60.
[12] Shih Fu Chang and David G. Messerschmitt, "Transform coding of arbitrarily-shaped image segments," in Proceedings of the First ACM International Conference on Multimedia, 1993, pp. 83-90.

[13] R.D. Dony and Simon Haykin, "Optimally adaptive transform coding," Image Processing, IEEE Transactions on, vol. 4, no. 10, pp. 1358-1370, Oct 1995.

[14] Y. Yamashita, "Image compression by weighted Karhunen-Loeve transform," in Pattern Recognition, 1996., Proceedings of the 13th International Conference on, Aug 1996, vol. 2, pp. 636-640 vol.2.

[15] T. Tanaka and Y. Yamashita, "A biorthogonal transform with overlapping and non-overlapping basis functions for image coding," Signal Processing, IEEE Transactions on, vol. 51, pp. 732-743, March 2003.

[16] M. Cagnazzo, R. Gaetano, S. Parrilli, and L. Verdoliva, "Region based compression of multispectral images by classified KLT," in Signal Processing Conference, 2006 14th European, Sept 2006, pp. 1-5.

[17] M. Cagnazzo, S. Parrilli, G. Poggi, and L. Verdoliva, "Improved class-based coding of multispectral images with shape-adaptive wavelet transform," Geoscience and Remote Sensing Letters, IEEE, vol. 4, no. 4, pp. 566-570, Oct 2007.

[18] M. Cagnazzo, G. Poggi, L. Verdoliva, and A. Zinicola, "Region-oriented compression of multispectral images by shape-adaptive wavelet transform and SPIHT," in Image Processing, 2004. ICIP '04. 2004 International Conference on, Oct 2004, vol. 4, pp. 2459-2462 Vol. 4.

[19] H. W. Kuhn, "The Hungarian Method for the assignment problem," Naval Research Logistics Quarterly, vol. 2, pp. 83-97, 1955.

[20] B. Penna, T. Tillo, E. Magli, and G. Olmo, "Transform coding techniques for lossy hyperspectral data compression," Geoscience and Remote Sensing, IEEE Transactions on, vol. 45, no. 5, pp. 1408-1421, May 2007. 